Nervenarzt 2012 $\cdot 83: 955-956$

DOI 10.1007/s00115-011-3461-x

Online publiziert: 18 . Juli 2012

(c) Springer-Verlag 2012

\title{
C. Gerloff
}

Abteilung Neurologie, Universitätsklinikum Hamburg-Eppendorf, Hamburg

Nichtmedikamentöse Therapien neurologischer Erkrankungen

Unser tägliches ärztliches Handeln ist geprägt durch Pharmakotherapien. Megatrials zu neuen Medikamenten mit 4- oder 5-stelligen Patientenzahlen gelten als Goldstandard - der „heilige Gral“ der evidenzbasierten Medizin. Neue Substanzen stammen dabei immer häufiger aus „High-throughput“-Verfahren, will heißen, eine vielversprechende Substanz wird auf mannigfaltigen „Essays“ getestet und zeigt dann mit etwas Glück den einen oder anderen pharmakodynamischen Effekt. Ein gutes Beispiel ist Levetiracetam. Es sollte eigentlich ein Antidementivum werden, zeigte dann aber überraschend gute antikonvulsive Effekte in den präklinischen Versuchen. Heute ist es einer der Blockbuster der Epileptologie.

Ganz anders ist der Entstehungsweg nichtmedikamentöser Therapien. Sie beruhen nahezu ausschließlich auf systematisch erarbeiteten pathophysiologischen Konzepten.

Zum Beispiel: Neurone funktionieren „elektrisch“, also liegt es nahe, nicht nur elektrische und magnetische Felder mittels Elektro- oder Magnetenzephalographie abzuleiten, sondern auch den umgekehrten Weg zu gehen und Neurone durch elektrische oder magnetische Stimulation zu manipulieren. Mittlerweile gibt es mehrere effektive Formen der Neurostimulation in diesem Sinne, so die transkranielle Gleichstromstimulation (tDCS), die transkranielle Magnetstimulation (TMS), aber auch die Vagusnervstimulation oder die Elektrostimulation des N. occipitalis major und natürlich die tiefe Hirnstimulation (THS; auch DBS für „deep brain stimulation“). Die- ses Themenheft wird den aktuellen Stand der Forschung und die Trends zur Neurostimulation nach Schlaganfall, Neurostimulation bei Kopfschmerzen, Depression, Epilepsie und Bewegungsstörungen zusammenfassen.

Was man heute als Science-Fiction beginnt, wird man morgen vielleicht als Reportage zu Ende schreiben müssen. (Norman Mailer, US-Schriftsteller, 1923-2007)

Die Zellen des Zentralnervensystems, ob Neurone, Gliazellen oder auch Tumorzellen oder Zellen in der Penumbra einer zerebralen Ischämie, zeichnen sich ganz offensichtlich durch bestimmte physikalische Eigenschaften aus, die ebenfalls Ansatzpunkte für nichtmedikamentöse Therapien bieten. Sie sind beispielsweise mehr oder weniger temperaturempfindlich oder reagieren unterschiedlich auf ein verändertes metabolisches Milieu. So können Tumorzellen durch erhöhte Temperaturen selektiv geschädigt werden. Andererseits können Neurone, die kritisch minderversorgt werden, durch Absenkung der Temperatur vor dem Zelltod geschützt werden. Kritische Stoffwechselsituationen sind möglicherweise auch durch das intelligente Zuführen von Energie wie $z$. B. durch Laserlicht zu beeinflussen. Tumorzellen mit hoher Zellteilungsrate sind hingegen empfindlich gegenüber hochfrequenten Wechselstromfeldern, weil diese mit Orientierung des Spindelapparates interferieren. Auch hieraus ergeben sich Ansatzpunkte für neue Behandlungskonzepte.

Je intensiver man sich mit dem Thema „nichtmedikamentöse Therapien“ in 
der Neurologie beschäftigt, desto mehr Facetten tun sich auf. Spannende Ansätze gibt es zu allen relevanten "großen“ Erkrankungsgruppen. Der Fokus dieses Themenheftes liegt auf Neurostimulationsverfahren in unterschiedlichsten Varianten, auf Neuromodulation, Neuroprotektion und auf Ansätzen, die das Stoffwechselmilieu von Zellen beeinflussen. Es muss an dieser Stelle ausdrücklich betont werden, dass natürlich neurochirurgische Operationen und neuroradiologische Interventionen zu den wichtigsten nichtmedikamentösen Therapien neurologischer Erkrankungen gehören und dementsprechend gut in der wissenschaftlichen Literatur repräsentiert sind. Und mehr noch: auch Physiotherapie, Logopädie, Ergotherapie, Neuropsychologie, Psychotherapie, aktivierende Pflege, sozialdienstliche Versorgung und Versorgung mit Orthesen und (Neuro-)Prothesen sind weitere Beispiele für wichtige nichtmedikamentöse Behandlungselemente, die - jede für sich - wiederum ein Themenheft füllen könnten und hier deshalb nicht mit abgehandelt werden.

Uns schien es wichtig, die neuen Entwicklungen im Bereich nichtmedikamentöser Therapien im genannten Sinne einmal zusammenzufassen und ich möchte die Gelegenheit nutzen, den Autoren der einzelnen Beiträge für ihr großes Engagement zu danken.

Ihnen, liebe Leser, wünsche ich eine informative und spannende Lektüre.

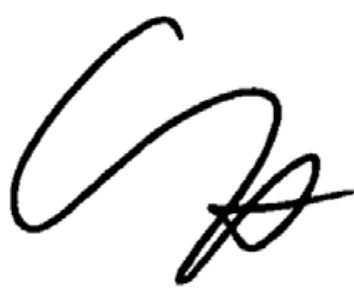

Prof. Dr. C. Gerloff

\section{Korrespondenzadresse}

Prof. Dr. C. Gerloff

Abteilung Neurologie, Universitätsklinikum Hamburg-Eppendorf

Martinistraße 52, 20246 Hamburg

gerloff@uke.de

Interessenkonflikt. Der korrespondierende Autor gibt an, dass kein Interessenkonflikt besteht.
M. Mäurer, M. Stangel (Hrsg.)

\section{Autoimmunerkrankungen in der Neurologie}

Diagnostik und Therapie

Berlin, Heidelberg, New York: Springer Verlag 2012, 277 S., 53 Abb., ISBN 978-3-642-20476-0, 99.00 EUR

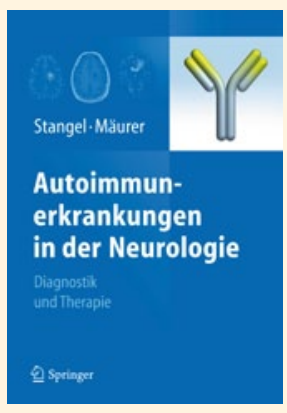

Die Multiple Sklerose ist zwar die häufigste neuroimmunologische Erkrankung, aber Neuroimmunologie ist weit mehr als nur Multiple Sklerose. Neuroimmunologie ist ein wesentliches

Teilgebiet der klinischen Neurologie, das neben den primären neurologischen Autoimmunerkrankungen des zentralen und peripheren Nervensystems auch eine Vielzahl internistischer Systemerkrankungen umfasst, die zu Läsionen und Funktionsstörungen des Nervensystems führen können. Diesem Umstand trägt die Monographie „Autoimmunerkrankungen in der Neurologie" von Stangel und Mäurer Rechnung: In 9 Kapiteln werden neben den klassischen neurologischen Autoimmunerkrankungen wie Multiple Sklerose, Guillain-Barré-Syndrom oder Myasthenia gravis auch internistische Systemerkrankungen wie die Vaskulitiden, Kollagenosen oder die Sarkoidose mit ihren spezifischen Auswirkungen auf das zentrale und periphere Nervensystem in einem einzigen Buch dargestellt. Das Werk zeichnet sich somit vor allem durch seinen interdisziplinären Ansatz aus, der sich in der Autorenliste aus Neurologen und Internisten widerspiegelt und der in dieser Form noch nicht in der deutschsprachigen Literatur existiert.

Das Buch wendet sich insbesondere an klinisch tätige Neurologen und Internisten und dient aufgrund des einheitlichen Aufbaus der Kapitel als zeitgemäßes und umfassendes Nachschlagewerk im Hinblick auf aktuelle diagnostische und therapeutische Konzepte. Es ist darüber hinaus jedoch auch eine angenehme Fortbildungslektüre, die den interessierten Leser mit wissenschaftlich fundierten Details zu den unterschiedlichen Autoimmunerkrankungen versorgt. Beispiel- haft herausgegriffen sei das Kapitel über die neurologischen Komplikationen entzündlicher Darmerkrankungen, das ausgezeichnet recherchiert ist und den Leser mit wichtigen Fakten im Hinblick auf differenzialdiagnostische Überlegungen versorgt.

Weniger gut ist die Bebilderung des $\mathrm{Bu}$ ches gelungen, insbesondere die Qualität der radiologischen Befunde lässt zu wünschen übrig. Darüber hinaus würde das Buch von einem Kapitel profitieren, in dem die wichtigsten Immuntherapien fachübergreifend dargestellt werden - v. a. im Hinblick auf den Wert des Buches als Nachschlagewerk. Insgesamt ist es jedoch ein sehr empfehlenswertes Buch - insbesondere für Neurologen und Internisten, die sich bereits schwerpunktmäßig mit dem Gebiet der Neuroimmunologie beschäftigen, aber auch für die, die sich diesem Feld kompetent und zeitgemäß nähern möchten.

H. Wiendl, Münster 